This document is the Accepted Manuscript version of a Published Work that appeared in final form in Dalton Transactions, copyright (C) Royal Society of Chemistry, after peer review and technical editing by the publisher.

To access the final edited and published work see Dalton Transactions 2020, 49, 6557-6560 https://doi.org/10.1039/D0DT01275K

Also see same web-link for Supporting Information, available free of charge. 


\section{Monofunctional Platinum(II) Anticancer Complexes Based on Multidentate Phenanthridine-Containing Ligand Frameworks}

Received 00th January 20xx, Accepted 00th January 20xx

DOI: $10.1039 / \times 0 \times x 00000 x$

\author{
Issiah B. Lozada, ${ }^{a}$ Bin Huang, ${ }^{a}$ Morgan Stilgenbauer, ${ }^{\mathrm{b}}$ Travis Beach, ${ }^{\mathrm{b}}$ Zihan Qiu, ${ }^{\mathrm{b}}$ Yaorong Zheng,
} David E. Herbert ${ }^{a,+}$
Phenanthriplatin is a leading preclinical anticancer Pt complex distinguished by a phenanthridine ligand that facilitates DNAtargeted covalent binding via intercalation. We report here that $\mathrm{Pt}$ (II) complexes incorporating phenanthridine into a chelating, multidentate ligand scaffold exhibit a superior in vitro therapeutic index compared with phenanthriplatin and cisplatin.

Cisplatin $^{1}$ and related platinum(II) drugs are key tools in modern cancer treatment. ${ }^{2,3}$ Notwithstanding its history of transformative clinical implementation, Pt chemotherapy can be limited by severe side effects caused by off-target activity and reduced efficacy due to acquired or intrinsic resistance in certain types of cancers. ${ }^{4}$ One strategy for increasing potency and expanding the spectrum of activity of a class of compounds while mitigating side effects is to search out analogues that operate by novel mechanisms of action. ${ }^{5}$ In this respect, monofunctional platinum anticancer complexes, a class of platin drug candidates containing only a single labile ligand first studied in earnest in the late 1980s, are attracting renewed interest. ${ }^{6}$ Compared with bifunctional anticancer complexes such as cisplatin which deform DNA strands via formation of inter- and intrastrand crosslinks, ${ }^{7}$ monofunctional Pt(II) complexes can only bind to DNA through a single coordination site opened up by the one vacating chloride. The antineoplastic activity of monofunctional complexes such as phenanthriplatin ([cis- $\mathrm{Pt}\left(\mathrm{NH}_{3}\right)_{2}$ (phenanthridine) $\left.\mathrm{Cl}\right]\left[\mathrm{NO}_{3}\right]$; Figure $1 \mathrm{a})^{8}$ thus arises from different biochemical interactions compared to compounds like cisplatin $\left(\right.$ cis- $\left.\mathrm{Pt}\left(\mathrm{NH}_{3}\right)_{2} \mathrm{Cl}_{2}\right)$, with a distinct spectrum of action and potential for altered resistance/side-effect profiles.

While phenanthriplatin shows heightened activity, ${ }^{8}$

\footnotetext{
a. Department of Chemistry and the Manitoba Institute for Materials, University of Manitoba, 144 Dysart Road, Winnipeg, Manitoba, R3T 2N2, Canada

${ }^{b .}$ Department of Chemistry, Kent State University, Kent, Ohio 44240, USA

† david.herbert@umanitoba.ca

Electronic Supplementary Information (ESI) available: full experimental details including NMR and IR spectra, supplementary assay plots and tables, and a combined crystallographic information file. CCDC Nos. 1959703-1959704 also contain the supplementary crystallographic data for this paper, which can be obtained free of charge from The Cambridge Crystallographic Data Centre via www.ccdc.cam.ac.uk/structures. See DOI: 10.1039/x0xx00000x
}

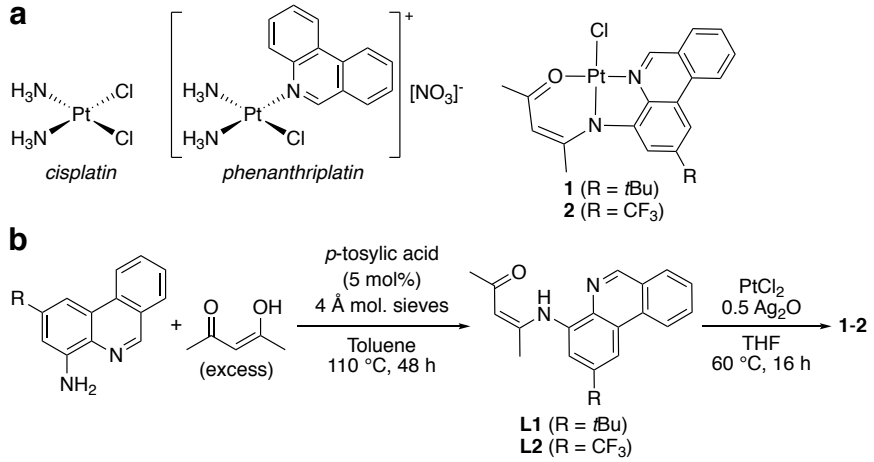

Figure 1. (a) Structures of cisplatin, phenanthriplatin ${ }^{8}$ and the multidentate phenanthridine-ligand supported Pt complexes described herein. (b) Synthesis of chelating $N^{\wedge} N(\mathrm{H})^{\wedge} O$ proligands L1-L2 and their Pt(II) complexes 1-2.

pyriplatin, in which phenanthridine is replaced with the parent $N$-heterocycle pyridine, is ten-fold less potent. ${ }^{9}$ Singlemolecule DNA-stretching experiments revealed a two-step binding process for phenanthriplatin, where rapid unwinding of DNA triggered by intercalation of the phenanthridine unit is followed by slower covalent modification. ${ }^{10}$ The smaller pyridine does not associate as effectively with duplex DNA prior to covalent binding, lowering efficacy. The disposition of the $\mathrm{N}$-heterocycle to the labile ligand is also important; DNA intercalation of the stereoisomer of phenanthriplatin with the heterocycle trans disposed to the chloride ([trans$\mathrm{Pt}\left(\mathrm{NH}_{3}\right)_{2}$ (phenanthridine) $\left.\left.\mathrm{Cl}\right]\left[\mathrm{NO}_{3}\right]\right)^{11}$ competes with - rather than enhancing - covalent binding, reducing the number of PtDNA adducts formed. ${ }^{10}$ Trans-phenanthriplatin is nevertheless still an effective anticancer agent, with quite different activity compared to phenanthriplatin. ${ }^{11}$ This is not true of transplatin. Covalent binding of phenanthridine to platinum to form a true monofunctional drug in phenanthriplatin also has superior activity compared with the simple combination of an intercalator such as ethidium bromide and cisplatin, which do not form a stable adduct in solution. ${ }^{12}$

We have recently developed synthetic mechanisms for incorporating phenanthridine into multidentate ligand architectures to explore their coordination chemistry with late 
transition metals. ${ }^{13-15}$ By appending additional donors, the heterocycle can be forced cis to the labile chloride and exhibits a diminished tendency to dissociate irreversibly from the metal thanks to the chelate effect. ${ }^{16,17}$ As the attenuation of chemical reactivity and possible side effects of bifunctional platin drugs such as carboplatin and oxaliplatin are attributed in part to the stabilizing impact of chelating ligand structures, ${ }^{18}$ we pursued the synthesis and characterization of multidentate phenanthridine-based ligands (L1 and L2) and their platinum complexes (1 and 2, Figure $\mathbf{1 b}$ ) to evaluate the potential of $\mathrm{Pt}$ (II) derivatives of chelating phenanthridine-based ligands as monofunctional chemotherapeutics. We find these chelatesupported phenanthriplatin analogs show a superior therapeutic index compared to cisplatin and phenanthriplatin in vitro.

Two $N^{\wedge} N(H)^{\wedge} O$ proligands containing phenanthridinyl units were prepared via acid-catalyzed condensation of 4aminophenanthridines $^{19}$ with acetylacetone (Figure 1b). The electronic influence of the substituent in the 2-position did not significantly influence the progress of the reaction. Proligands bearing electron-releasing tBu (L1) and electron-withdrawing $\mathrm{CF}_{3}$ substituents ( $\mathbf{L 2}$ ) could be isolated in similar yields ( $\left.65 \%\right)$. Single crystals of L1 suitable for X-ray crystallography were grown from mixtures of diethylether and chloroform (Figure 2a). The structural metrics are consistent with a keto/enamine tautomer. In particular, the solid-state structure revealed a short C(21)-O(1) bond distance of 1.244(3) $\AA$. This assignment was corroborated by comparing solution $N M R$ and IR parameters with related compounds. ${ }^{20}$ Density functional theory (DFT; RIJCOSX-PBEO/def2-TZVP) predicted IR spectra of the optimized structures of $\mathbf{L} \mathbf{1}$ and $\mathbf{L} \mathbf{2}$ accordingly reproduce the two notable absorptions observed experimentally between $1550-1650 \mathrm{~cm}^{-1}$. The medium-strength, narrow peaks at 1617 $\mathrm{cm}^{-1}$ (L1) and $1634 \mathrm{~cm}^{-1}$ (L2) are consistent with C=O stretching modes, while the stronger absorptions at $1570 \mathrm{~cm}^{-1}$ (L1) and $1579 \mathrm{~cm}^{-1}$ (L2) are attributed to $\mathrm{N}-\mathrm{H}$ bends.

Metallation of the proligands was carried out using $\mathrm{PtCl}_{2}$ in the presence of 0.5 equivalents of silver oxide in THF at elevated temperatures. Again, the electronics of the phenanthridinyl unit did not impact the progress of the reaction. Platinum complexes $\mathbf{1}$ and $\mathbf{2}$ were isolated as airstable orange solids in similar yields ( 86-87\%). Ligand binding was confirmed by disappearance of the downfield ${ }^{1} \mathrm{H}$ NMR signal attributed to the acidic $\mathrm{N}-\mathrm{H}$ proton of the proligands (L1: 13.44 ppm; L2: $13.72 \mathrm{ppm}$ ) and a shift in the $\mathrm{CH}$ resonance in the 6-position of the phenanthridinyl ring system, which shows coupling to the ${ }^{195} \mathrm{Pt}$ nuclei in $\mathbf{1}$ and $\mathbf{2}$ (1: $10.03 \mathrm{ppm},{ }^{3} \mathrm{~J}_{\mathrm{PtH}}=39$ $\mathrm{Hz}$; $2: 10.20 \mathrm{ppm},{ }^{3} J_{\mathrm{PtH}}=39 \mathrm{~Hz}$ ). A similar deshielding of this particular hydrogen nucleus was observed for complexes of bis(phenanthridinyl)amido ligands ${ }^{16}$ and can be interpreted as diagnostic of phenanthridinyl binding to a late transition metal. Preparations using oxygenous or nitrogenous Brönsted bases (e.g., $\mathrm{NEt}_{3}$ or $\mathrm{NaOtBu}$ ) in place of $\mathrm{Ag}_{2} \mathrm{O}$ were similarly successful in generating the target platinum(II) complexes. Single-crystals suitable for crystallographic analysis of $\mathbf{1}$ were also obtained. The solid-state structure (Figure $2 \mathrm{~b}$ ) reveals the co-planarity of the phenanthridinyl moiety and the
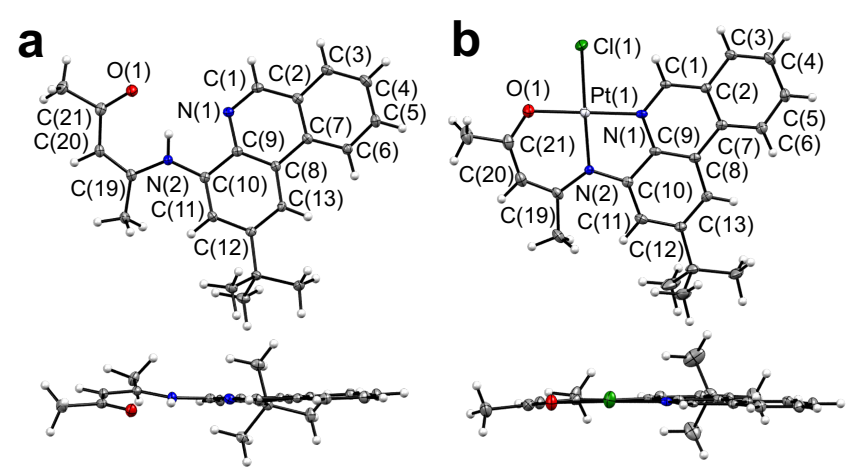

Figure 2. Two views of the solid-state structure of (a) $\mathbf{L} \mathbf{1}$ and (b) $\mathbf{1}$ with ellipsoids shown at $30 \%$ probability. Selected bond distances $(\AA)$ : L1: C(1)-N(1) 1.303(3), $\mathrm{C}(9)-\mathrm{N}(1) 1.380(3), \mathrm{C}(10)-\mathrm{N}(2) 1.396(3), \mathrm{C}(19)-\mathrm{N}(2)$ 1.363(3), C(19)-C(20) 1.370(3), $\mathrm{C}(20)-\mathrm{C}(21)$ 1.433(3), $\mathrm{C}(21)-\mathrm{O}(1)$ 1.244(3). 1: $\mathrm{N}(1)-\mathrm{Pt}(1)$ 1.978(4), N(2)-Pt(1) 1.991(3), (0)-Pt(1) 1.979(3), Cl(2)-Pt(1) 2.3137(12), C(10)-N(2) 1.419(6), C(19)-N(2) 1.357(6), $C(19)-C(20) 1.402(7), C(20)-C(21)$ 1.376(8), $C(21)-O(1) 1.278(6) ; N(1)-$ $\mathrm{Pt}(1)-\mathrm{N}(2)$ 82.68(14), N(1)-Pt-(1)O(1) 178.49(15), N(1)-Pt(1)Cl(2) 95.34(10), N(2)$\mathrm{Pt}(1)-\mathrm{O}(1)$ 97.61(15), N(2)-Pt(1)-Cl(1) 176.70(11), O(1)-Pt(1)-Cl(2) 84.44(11).

coordination plane of platinum, with an angle between the two planes of $3.5^{\circ}$. The short $C(21)-O(1)$ of $1.278(6) \AA$ is consistent with retention of the keto/enamide structure upon coordination to Pt. Complexes $\mathbf{1}$ and $\mathbf{2}$ are generally soluble in organic solvents, though insoluble in aqueous media.

To assess the biological potential of chelated phenanthridine-containing monofunctional Pt(II) compounds $\mathbf{1}$ and $\mathbf{2}$, in vitro cytotoxicities were evaluated using MTT assays (MTT $=$ [3-(4,5-dimethylthiazol-2-yl)-2,5-diphenyltetrazolium bromide]; see Supporting Information). Table S1 reports $\mathrm{IC}_{50}$ (50\% growth inhibition concentrations) for two separate ovarian cancer cell lines. The results revealed promising activity for both $\mathbf{1}$ and $\mathbf{2}$ compared with cisplatin, as well as a dependence on substituent structure. For example, $\mathbf{2}\left(\mathrm{R}=\mathrm{CF}_{3}\right)$ showed much higher in vitro efficacy as compared to cisplatin than $1(\mathrm{R}=t \mathrm{Bu})$, as well as less resistance than cisplatin (IC $\mathrm{I}_{50}$ of A2780cis/IC50 of A2780) against both A2780 (cisplatin sensitive) and $A 2780$ cis (cisplatin resistant) ovarian cancer cell lines. The higher in vitro efficacy of $\mathbf{2}\left(\mathrm{R}=\mathrm{CF}_{3}\right)$ vs $\mathbf{1}(\mathrm{R}=t \mathrm{Bu})$ highlights the opportunity to fine-tune biological activity via ligand backbone substitution.

In addition, neither the proligand $\mathbf{L} \mathbf{2}$ or precursors 4-amino(2-tert-butyl)phenanthridine or 4-amino-(2-trifluoromethyl)phenanthridine (which may be generated upon hydrolysis of L1 or L2) were found to be effective in the absence of Pt(II). The differing profile compared to cisplatin (i.e., higher in vitro efficacy and lower cross-resistance) implies a different mechanism of operation from cisplatin, which, considering the planar structure of $\mathbf{1}$ and $\mathbf{2}$ compared to phenanthriplatin ${ }^{8}$ may involve a more prominent role for intercalation. As noted above, intercalation enhances covalent binding and ultimately boosts the number of complex-DNA adducts observed for phenanthriplatin, but only when these two processes are concurrent. ${ }^{10}$ The lack of activity in the absence of $\mathrm{Pt}(\mathrm{II})$ highlights a key role for the metal centre in the cytotoxicity of 1 and 2. Phenanthridines in general are anticipated to interact with DNA via an intercalation mechanism, similar to the 
a

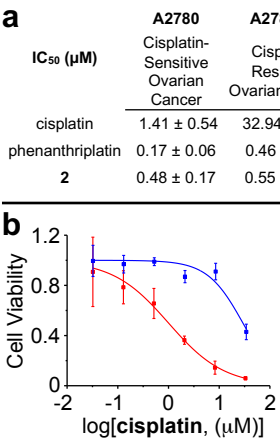

MET5A HEK-293

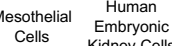
Cells Kidney Cells $4.99 \pm 0.87$ 2.18

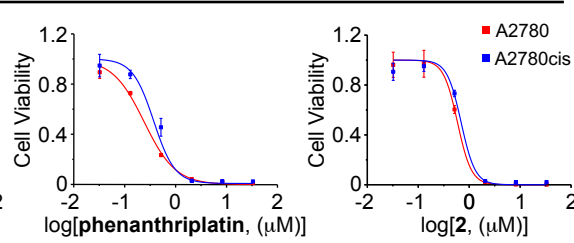

Figure 3. Cytotoxicity profiles of cisplatin, phenthriplatin, and $\mathbf{2}$ against a panel of human cancer and normal cell lines: (a) $I_{50}$ values, and (b) killing curves of cisplatin, phenanthriplatin, and $\mathbf{2}$ against $\mathrm{A} 2780$ and $\mathrm{A} 2780$ cis ovarian cancer cells highlighting the lower resistance factor (RF) of $\mathbf{2}$.

mechanism of operation of the DNA stain ethidium bromide, of which phenanthridine forms the molecular core. ${ }^{21}$ The poor activity of the aminophenanthridines excludes demetallation or hydrolysis of decoordinated proligands as the source of the observed in vitro anticancer activity.

With these results in hand, complex 2 was selected for further screening against additional human cancer and noncancerous cell lines, including the non-small cell lung cancer cell line A549, ovarian cancer cell line A2780, and cisplatinresistant ovarian cancer cell line A2780cis, ovarian cancer cell line SKOV-3, triple-negative breast cancer cell line MDA-MB231, non-cancerous mesothelial cell line MET-5A and noncancerous kidney cell line HEK293. Cisplatin and phenanthriplatin were used as controls. Cancerous and normal cells were treated for $72 \mathrm{~h}$ and cell viability was assessed. The $\mathrm{IC}_{50}$ values represent compound concentrations required to inhibit cell growth by $50 \%$, and these data are tabulated in Figure 3a. Compared with cisplatin, $\mathbf{2}$ exhibits much lower $I C_{50}$ values among all tested cell lines. For example, in A2780cis cisplatin-resistant ovarian cancer cell line, the $I C_{50}(2)=$ $0.55 \pm 0.22 \mu \mathrm{M}$ is nearly 60 times lower than that of cisplatin $\left(\mathrm{IC}_{50}=32.94 \pm 8.82 \mu \mathrm{M}\right)$.

Complex 2 also has comparable efficacy to phenanthriplatin across a range of cancer cell lines under the conditions tested. Importantly, however, $\mathbf{2}$ proved less toxic to normal cells (MET-5A and HEK293) compared with phenanthriplatin and displays a lower resistance factor ( $R F=$ $I C_{50 \text { (A2780cis) }} / I_{C_{50(A 2780)}}=1.1$ ) in ovarian cancer cell lines than cisplatin ( $R F=23$ ) or phenanthriplatin ( $R F=2.7$; Figure $3 b)$. The MTT results collectively support that the chelated monofunctional phenanthridine-based platinum compound $\mathbf{2}$ shows a superior therapeutic index compared with cisplatin and phenanthriplatin in vitro.

We next investigated the cellular uptake and cellular responses of 2. First, uptake was evaluated using graphite furnace atomic absorption spectroscopy (GFAAS), with cisplatin and phenanthriplatin again employed as controls. SKOV 3 cells were incubated for $24 \mathrm{~h}$ with $2 \mu \mathrm{M}$ concentrations of each of the three different platinum compounds. The treated cells were then harvested and digested for GFAAS analysis. Complex 2 exhibits higher cellular uptake (4.09 \pm 0.138 pmol Pt per million cells) compared with cisplatin $(2.12$ \pm 0.129 pmol Pt per million cells) or phenthriplatin $(2.88 \pm$
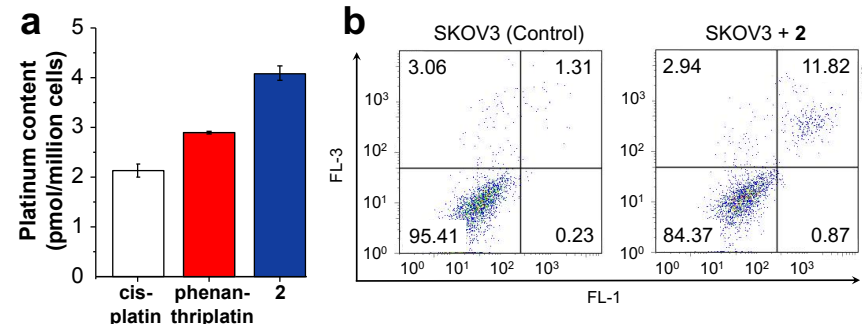

Figure 4. (a) Cellular uptake of cisplatin, phenthriplatin, and $\mathbf{2}$ in SKOV3 ovarian cancer cells ([Pt $]=2 \mu \mathrm{M}, 24 \mathrm{~h}$ at $\left.37{ }^{\circ} \mathrm{C}, 5 \% \mathrm{CO}_{2}\right)$; (b) Annexin V/PI flow cytometric analysis of the apopotic events of SKOV3 cells with or without the treatment of $\mathbf{2}$ $\left([\mathrm{Pt}]=1 \mu \mathrm{M}, 72 \mathrm{~h}\right.$ at $\left.37^{\circ} \mathrm{C}, 5 \% \mathrm{CO}_{2}\right)$.

0.023 pmol Pt per million cells; Figure 4a). Similar to cisplatin, ${ }^{22}$ phenanthriplatin uptake has been shown to be mediated by organic cation transporters (OCT); phenanthriplatin is considered a high affinity substrate for OCT2, while showing a lower apparent affinity for the multi-drug and toxin extrusion proteins (MATE) responsible for excretion of platinum into the urine. $^{23}$ Though not a cation itself, a similar affinity for transport and extrusion proteins might be plausibly expected for the chemically related $\mathbf{2}$, as also has been observed for initially neutral platins such as cisplatin and oxaliplatin. ${ }^{24}$ The enhanced uptake of $\mathbf{2}$ compared with phenanthriplatin does not clearly correlate with decreased cell viability for SKOV3 cells. This effect plausibly also features in the lower toxicity observed in vitro towards non-cancer cell lines.

With respect to cellular responses, a dual staining Annexin V/PI flow cytometry assay was used to probe the occurrence of apoptosis. In particular, SKOV3 ovarian cancer cells were treated with and without 2 . The results in Figure $4 \mathrm{~b}$ clearly indicate that $\mathbf{2}$ induced apoptosis, stimulating SKOV3 cells to undergo early $(0.87 \%)$ and late $(11.82 \%)$ stage apoptosis after $72 \mathrm{~h}$ of incubation, the populations of which were much higher than those of control. The evidence compiled from the cellbased studies suggest that planar phenanthridine-ligated Pt(II) complexes such as $\mathbf{2}$ can readily enter cancer cells and trigger apoptosis.

Monofunctional phenanthriplatin-type complexes based on chelating tridentate $N$-heterocycle-containing ligands thus show promising in vitro anticancer activity, highlighting the potential of this new class of anticancer agents. The high activity towards cisplatin-resistant cancer cells is a critical finding, as tumors resistant to cisplatin often show crossresistance to a diverse range of unrelated antitumour drugs. ${ }^{25}$ The activation of independent pathways by the molecular structure of phenanthridine-based $\mathrm{Pt}$ (II) complexes similar to what is observed with phenanthriplatin ${ }^{8}$ is likely responsible for the increased sensitivity of resistant cells to $\mathbf{1}$ and $\mathbf{2}^{26}$

In addition, a distinguishing feature of both cisphenanthriplatin and trans-phenanthriplatin is the orientation of the phenanthridine ligand with respect to the coordination plane of platinum. The heterocycle is nearly orthogonal in the cis isomer (dihedral angle $\left.\sim 89^{\circ}\right)^{8}$ and slightly less so in transphenanthriplatin $\left(\sim 67^{\circ}\right) .{ }^{11}$ Coupled with the asymmetry of phenanthridine with respect to the position of benzannulation relative to the nitrogen atom, phenanthriplatin is chiral. ${ }^{27}$ While racemization upon rotation about the Pt$\mathrm{N}$ (phenanthridine) bond is rapid enough to preclude requiring 
administration of a single enantiomer, there is a preference for diastereomer formation upon binding to DNA. ${ }^{27}$ Forcing the phenanthridinyl unit coplanar with the metal coordination plane obviates this chirality and raises the interesting question of why $\mathbf{1}$ and $\mathbf{2}$ show enhanced anticancer efficacy compared with phenanthriplatin in vitro. Identification of the molecular targets of $\mathbf{1}$ and $\mathbf{2}$ and investigation of potential intercalationbased mechanisms ${ }^{28}$ therefore represent the next steps in this line of inquiry.

We are grateful for support from Research Manitoba (New Investigator Grant to DEH), the Canadian Foundation for Innovation (CFI \#32146); the University of Manitoba for GETS support, the Bert \& Lee Friesen Graduate Scholarship (IBML), and a Faculty of Science Undergraduate Summer Research Award (BH); and to S. McKenna and G. Tranmer for helpful discussions.

\section{Conflicts of interest}

There are no conflicts to declare.

\section{References}

1 B. Rosenberg, L. VanCamp, J. E. Trosko and V. H. Mansour, Nature, 1969, 222, 385-386.

2 D. Wang and S. J. Lippard, Nat. Rev. Drug Discov., 2005, 4, 307-320.

3 B. Englinger, C. Pirker, P. Heffeter, A. Terenzi, C. R. Kowol, B. K. Keppler and W. Berger, Chem. Rev., 2019, 119, 1519-1624.

4 N. J. Wheate, S. Walker, G. E. Craig and R. Oun, Dalton Trans., 2010, 39, 8113-8127.

5 T. C. Johnstone, G. Y. Park and S. J. Lippard, Anticancer Res., 2014, 34, 471-476.

6 T. C. Johnstone, J. J. Wilson and S. J. Lippard, Inorg. Chem., 2013, 52, 12234-12249.

7 R. C. Todd and S. J. Lippard, J. Inorg. Biochem., 2010, 104, 902-908.

8 G. Y. Park, J. J. Wilson, Y. Song and S. J. Lippard, Proc. Natl. Acad. Sci. U.S.A., 2012, 109, 11987-11992, S11987/11981S11987/11924.

9 K. S. Lovejoy, M. Serova, I. Bieche, S. Emami, M. D'Incalci, M. Broggini, E. Erba, C. Gespach, E. Cvitkovic, S. Faivre, E. Raymond and S. J. Lippard, Mol. Cancer Ther., 2011, 10, 1709-1719.

10 A. A. Almaqwashi, W. Zhou, M. N. Naufer, I. A. Riddell, Ö. H. Yilmaz, S. J. Lippard and M. C. Williams, J. Am. Chem. Soc., 2019, 141, 1537-1545.

11 W. Zhou, M. Almeqdadi, M. E. Xifaras, I. A. Riddell, Ö. H. Yilmaz and S. J. Lippard, Chem. Commun., 2018, 54, 27882791.

12 T. D. Tullius and S. J. Lippard, Proc. Natl. Acad. Sci. U.S.A., 1982, 79, 3489-3492.

13 R. Mondal, P. K. Giesbrecht and D. E. Herbert, Polyhedron, 2016, 108, 156-162.

14 R. Mondal, I. B. Lozada, R. L. Davis, J. A. G. Williams and D. E. Herbert, Inorg. Chem., 2018, 57, 4966-4978.

15 I. B. Lozada, T. Murray and D. E. Herbert, Polyhedron, 2019, 161, 261-267.

16 P. Mandapati, P. K. Giesbrecht, R. L. Davis and D. E. Herbert, Inorg. Chem., 2017, 56, 3674-3685.

17 P. Mandapati, J. D. Braun, C. Killeen, R. L. Davis, J. A. G. Williams and D. E. Herbert, Inorganic Chemistry, 2019, 58, 14808-14817.
18 K. D. Mjos and C. Orvig, Chem. Rev., 2014, 114, 4540-4563.

19 J. D. Braun, I. B. Lozada, C. Kolodziej, C. Burda, K. M. E. Newman, J. van Lierop, R. L. Davis and D. E. Herbert, Nat. Chem., 2019, 11, 1144-1150.

20 R. A. M. O'Ferrall and B. A. Murray, J. Chem. Soc., Perkin Trans. 2, 1994, 2461-2470.

21 P. J. B. Le, Methods Biochem Anal, 1971, 20, 41-86.

22 K. K. Filipski, W. J. Loos, J. Verweij and A. Sparreboom, Clin. Cancer Res., 2008, 14, 3875-3880.

23 A. Hucke, G. Y. Park, O. B. Bauer, G. Beyer, C. Koeppen, D. Zeeh, C. A. Wehe, M. Sperling, R. Schroeter, M. Kantauskaite, Y. Hagos, U. Karst, S. J. Lippard and G. Ciarimboli, Front. Chem., 2018, 6, 180/181-180/189.

24 H. Burger, W. J. Loos, K. Eechoute, J. Verweij, R. H. J. Mathijssen and E. A. C. Wiemer, Drug Resist. Updates, 2011, 14, 22-34.

25 R. F. Ozols, Hematol. Oncol. Clin. N. Am., 6, 879-894.

26 Z. H. Siddik, Oncogene, 2003, 22, 7265-7279.

27 T. C. Johnstone and S. J. Lippard, J. Am. Chem. Soc., 2014, 136, 2126-2134.

28 E. Y. D. Chua, G. E. Davey, C. F. Chin, P. Dröge, W. H. Ang and C. A. Davey, Nuc. Acids Res., 2015, 43, 5284-5296. 